\title{
IONIC FRAGMENTATION OF NO FOLLOWING EXCITATION OF THE NK-SHELL AND THE OK-SHELL ELECTRON
}

\author{
ISAO H. SUZUKI and NORIO SAITO \\ Electrotechnical Laboratory, Umezono, Tsukuba-shi, Ibaraki, 305 Japan
}

(Received May 21, 1994)

\begin{abstract}
Ionic fragmentation of NO stimulated by soft X-ray absorption has been studied using a monochromatized synchrotron radiation and a time-of-flight mass spectrometer. In photoexcitation of a $1 \mathrm{~s}$ electron, the singly charged molecular ion $\mathrm{NO}^{+}$was formed only at 410 and $533 \mathrm{eV}$ (transitions to the $2 \pi$ orbital), and a fragment ion $\mathrm{N}^{+}$had the highest intensity in all the energies. The doubly charged molecular ion was produced appreciably, and fragment ions $\left(\mathrm{N}^{2+}\right.$ and $\left.\mathrm{O}^{2+}\right)$ were formed considerably even below the $1 \mathrm{~s}$ ionization thresholds. The measured time-of-flight spectra were converted into kinetic energy distributions of $\mathrm{N}^{+}, \mathrm{O}^{+}, \mathrm{N}^{2+}$ and $\mathrm{O}^{2+}$ at photon energies for characteristic excitation by a simulation calculation. The dissociation pathways from the core-hole states of NO were discussed using the kinetic energy distribution and ion intensity ratios as well as Auger electron spectra in the literature.
\end{abstract}

KEY WORDS: Ionic fragmentation, Inner-shell excitation, Kinetic energy release, Doubly charged NO, TOF mass spectrometer, $\pi^{*}$ states.

\section{INTRODUCTION}

There have been numerous studies on the dissociative ionization of NO. ${ }^{1-10}$ Branching ratios for produced ions and appearance potentials of fragment ions were utilized for discussion on various intermediate states in dissociation processes. Using simulated photoionization techniques, $(\mathrm{e}, 2 \mathrm{e})$ and $(\mathrm{e}, \mathrm{e}+\mathrm{ion})$, Iida and coworkers have determined absolute partial oscillator strength for photoionization of individual ionic states and for formation of individual fragment ions in the region of vacuum ultraviolet radiation up to $80 \mathrm{eV}$. They proposed a breakdown scheme to $\mathrm{N}^{+}, \mathrm{O}^{+}$and $\mathrm{NO}^{2+}$ based on their experimental results. Samson et al. obtained partial photoionization cross sections for $\mathrm{N}^{+}, \mathrm{O}^{+}$and $\mathrm{NO}^{2+}$ and discussed the dissociation pathways from $\mathrm{c}^{3} \Pi$ and $\mathrm{B}^{\prime 1} \Sigma^{+}$states of $\mathrm{NO}^{+}{ }^{5}$ Carlson and Krause measured fragment ions formed from NO following absorption of characteristic soft X-rays, which showed the difference of ion yield ratios between valence electron ionization and inner-shell excitation. ${ }^{8}$ A number of research groups have observed inner-shell excitation spectra using monochromatized synchrotron radiation and an electron energy loss technique. ${ }^{11-15}$ The most prominent feature is a strong peak at $399.7 \mathrm{eV}$, which is assigned 
to the transition of N1s $\rightarrow 2 \pi$ orbital $\left(\pi^{*}\right)$. The recent studies with high resolution monochromators clarified that this peak is composed of ${ }^{2} \Delta,{ }^{2} \Sigma^{-}$and ${ }^{2} \Sigma^{+}$states including some vibrational excitations from lower energy to higher. ${ }^{12,13}$ The counterpart of this transition in the OK-edge region showed a broader peak of $2.5 \mathrm{eV}$ width, $1 \sigma^{1} 2 \pi^{2}$, but the order of the states, ${ }^{2} \Sigma^{-},{ }^{2} \Delta$ and ${ }^{2} \Sigma^{+}$, was found to differ from that at the NK-edge. The inner-shell spectra exhibited small structures in the Rydberg excitation region and a broad intensity increase at the $\sigma$-type shape resonance above ionization thresholds.

After the inner-shell excitation, the produced inner-hole is usually filled with a valence electron through Auger decay because soft X-ray emission occurs only with a negligibly low probability. The molecular ion in a highly excited state decomposes to ionic fragments. Inner-shell excitation makes a hole at a localized site in a molecule, which frequently induces an Auger transition pertaining to the orbital localized to this site. Thus site specific fragmentation has been expected to take place in the molecule if this molecule is excited to some inner-shell hole state. ${ }^{16-18}$ Fragmentation of several polyatomic molecules was studied using monochromatized synchrotron radiation, which yielded specific fragmentation patterns for ionic products. In instances of diatomic molecules like NO, the number of ionic products is limited. Although the valence electron orbital is not much localized at the atomic site, the charge distribution of valence holes generated through the Auger decay has a possibility to be different in the N1s and O1s Auger processes. This difference is presumed to induce different yield ratios for fragment ion formation. According to previous studies on Auger electron spectra, the final states of $\mathrm{NO}^{2+}$ are appreciably different in the two different hole states. ${ }^{19-22}$

Fragment ions produced were found to have some kinetic energies in the inner valence ionization and also in soft X-ray excitation. . $, 5,8-10$ As mentioned above, two ionic fragments are usually generated in the inner-shell excitation, and thus fragment ions have often a significantly larger kinetic energies than the valence electron ionization, owing to Coulomb repulsion force. , $^{8,18,23,24}$ It is important to obtain kinetic energy distributions of fragment ions from several inner-shell excited states as well as branching ratios of ion yields, in order to clarify characteristics of the site specific fragmentation.

In the present study, the ions from NO induced through excitation of N1s and O1s electrons have been measured using synchrotron radiation and a time-of-flight (TOF) mass spectrometer. The measured TOF spectra were reproduced by a simulation fitting calculation, which provided kinetic energy distributions of $\mathrm{N}^{+}, \mathrm{O}^{+}, \mathrm{N}^{2+}$ and $\mathrm{O}^{2+}$. The dissociation pathways are discussed on the basis of the present results and the literature data.

\section{EXPERIMENTAL}

Synchrotron radiation from TERAS (an electron storage ring at the Electrotechnical Laboratory) was collected with a prefocusing mirror system, dispersed by a Grasshopper monochromator (grating radius $=2 \mathrm{~m}$, grating groove density $=2400 \mathrm{1} / \mathrm{mm}$ ), 
and reflected into the horizontal direction with a refocusing mirror. ${ }^{25,26}$ The monochromatized synchrotron radiation (energy spread $=0.8 \mathrm{eV}$ at the $\mathrm{NK}$-edge and $1.6 \mathrm{eV}$ at the OK-edge) entered the chamber including a time-of-flight (TOF) mass spectrometer. The detail of the experimental arrangement and the TOF measurement was described elsewhere. ${ }^{26}$ Briefly, the photon beam crossed perpendicularly an effusive beam of NO. The electron produced was extracted in the direction perpendicular to both the photon and molecular beams. The ions produced were extracted in the direction opposite to that of electron extraction. Both charged species were detected with microchannel plates (MCP). A TOF spectrum of the ions was observed using the electron signal as a start pulse. The angle between the direction of photon polarization and the axis of the TOF spectrometer was set at $54.7^{\circ}$, because the dissociation of molecular ions often occurs with an anisotropic distribution. ${ }^{11,12,25,27}$

When the TOF spectrum was measured in detail at fixed incident photon energies, a lower electric field, $13 \mathrm{~V} / \mathrm{mm}$, was applied to the TOF spectrometer. The measured TOF spectra were reproduced by a simulation calculation, the procedure of which was reported previously. ${ }^{11,23-28}$ Concisely, the main assumptions are the following: (1) Real electric fields are identical to those designed. (2) Space focusing of the TOF spectrometer is perfect. (3) Target gas density in the collision region is uniform. Then, the trajectory of the ion formed with a certain kinetic energy was calculated inside the TOF spectrometer. This trajectory calculation gave us the flight time of the ion and its collection efficiency in the MCP detecter.

\section{RESULTS AND DISCUSSION}

\section{A. Ion yield spectra}

When intensities of individual ions were measured as a function of photon energy, the applied electric field in the TOF mass spectrometer was set to be $26 \mathrm{~V} / \mathrm{mm}$. Figure 1 shows the energy dependence of these ion yields in the region of the nitrogen $\mathrm{K}$-edge, together with the summation of these ion yields and the electron yield. The bars with hatching denote the ionization thresholds, ${ }^{3} \Pi$ at $410.3 \mathrm{eV}$ and ${ }^{1} \Pi$ at $411.8 \mathrm{eV}$. The most prominent peak at $399.7 \mathrm{eV}$ in all yield curves originates from the transition from the $2 \sigma$ orbital (N1s) to the $2 \pi$ orbital $\left(\pi^{*}\right)$, as previously shown. ${ }^{11-15}$ The electron yield spectrum is close to the photoabsorption cross section, which indicates the strong peak of the $2 \sigma$ to $2 \pi$ transition, small structures of Rydberg orbital excitation at $406-409 \mathrm{eV}$, and intensity increase at the ionization threshold followed by broad structures of the $\sigma$-type shape resonance.

The total ion spectrum shows a similar feature to the electron yield, but the shape of the total ion above $406 \mathrm{eV}$ is slightly different from the electron. The structures of the Rydberg orbital transition in the total ion curve are not clearer than the electron spectrum.

The yield spectrum of $\mathrm{NO}^{+}$is much different from other curves except the strong peak at $399.7 \mathrm{eV}$. The yield of $\mathrm{NO}^{+}$shows a constant intensity in these energies above the position of this peak, suggesting that $\mathrm{NO}^{+}$is not produced via inner-shell 


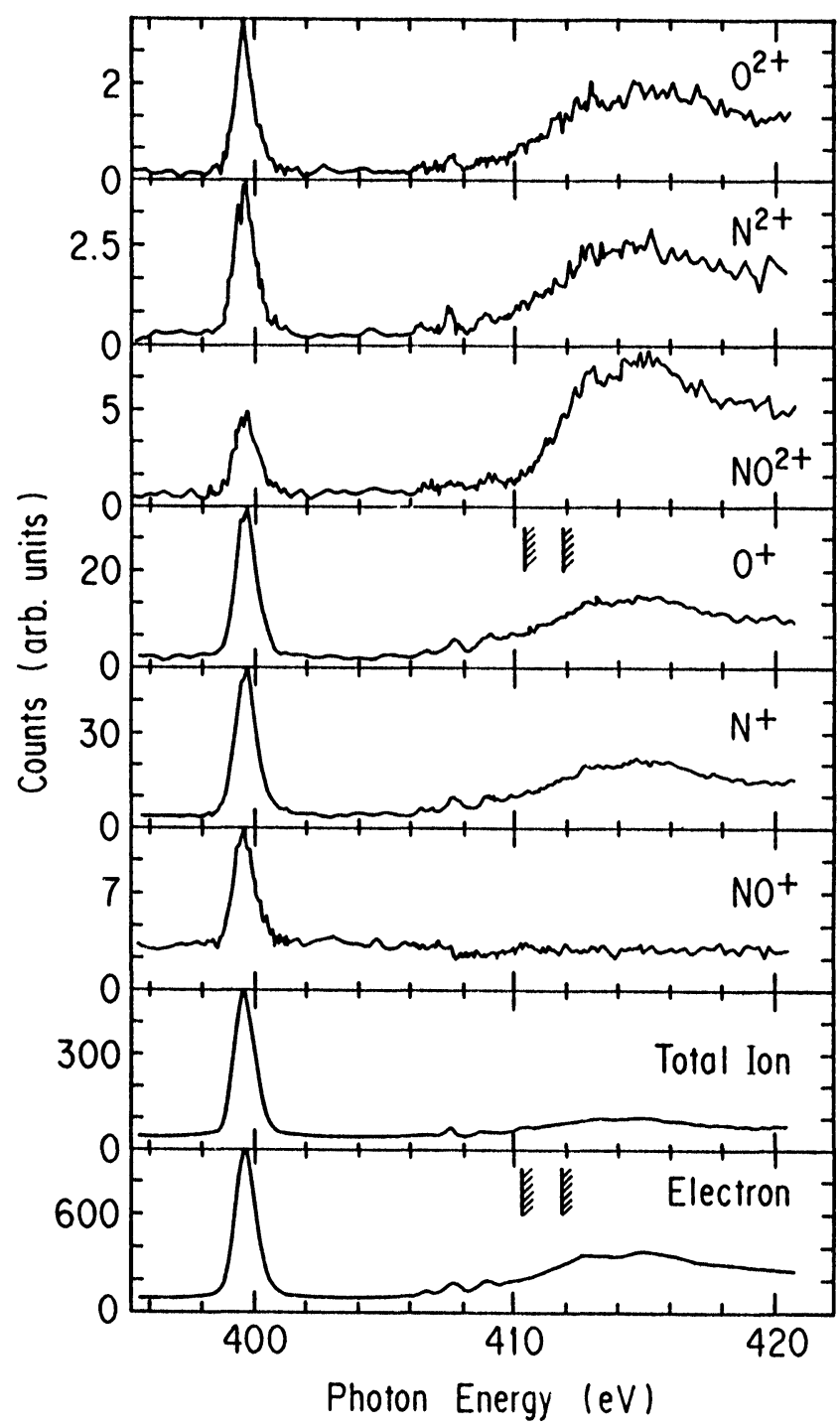

Figure 1 Yield spectra of individual ions produced from NO around the NK-edge, together with the total ion and the electron. Bars with hatching denote the ionization thresholds, ${ }^{3} \Pi$ at $410.3 \mathrm{eV}$ and ${ }^{1} \Pi$ at $411.8 \mathrm{eV}$.

excitation above $405 \mathrm{eV}$. The yield curves for $\mathrm{N}^{+}$and $\mathrm{O}^{+}$have profiles close to the electron yield, but the relative intensities above the Rydberg orbital excitation are higher than that of the electron in comparison with those of the most prominent peak at $399.7 \mathrm{eV}$. Those for $\mathrm{N}^{2+}$ and $\mathrm{O}^{2+}$ show a similar spectrum to the electron but the relative intensities indicate a larger increase above the ionization thresholds than those for $\mathrm{N}^{+}$and $\mathrm{O}^{+}$. The curve for the doubly charged molecular ion $\left(\mathrm{NO}^{2+}\right)$ exhibits this intensity change most distinctly, that is, the intensities above the thresholds are 
higher than that of the $2 \sigma$ to $2 \pi$ transition. These phenomena suggest that branching ratios of ion production vary with the photon energy and that the probability of the double ionization increase with increasing photon energy.

The yield spectra of ions produced from NO in the region of the OK-edge are shown in Fig. 2, as well as the yield of the total ion and the electron. The bars with hatching denote the ionization thresholds of the OK-shell, ${ }^{3} \Pi$ at $543.3 \mathrm{eV}$ and ${ }^{\prime} \Pi$ at $543.8 \mathrm{eV}$. The yield spectra of the total ion and of the electron are similar to the

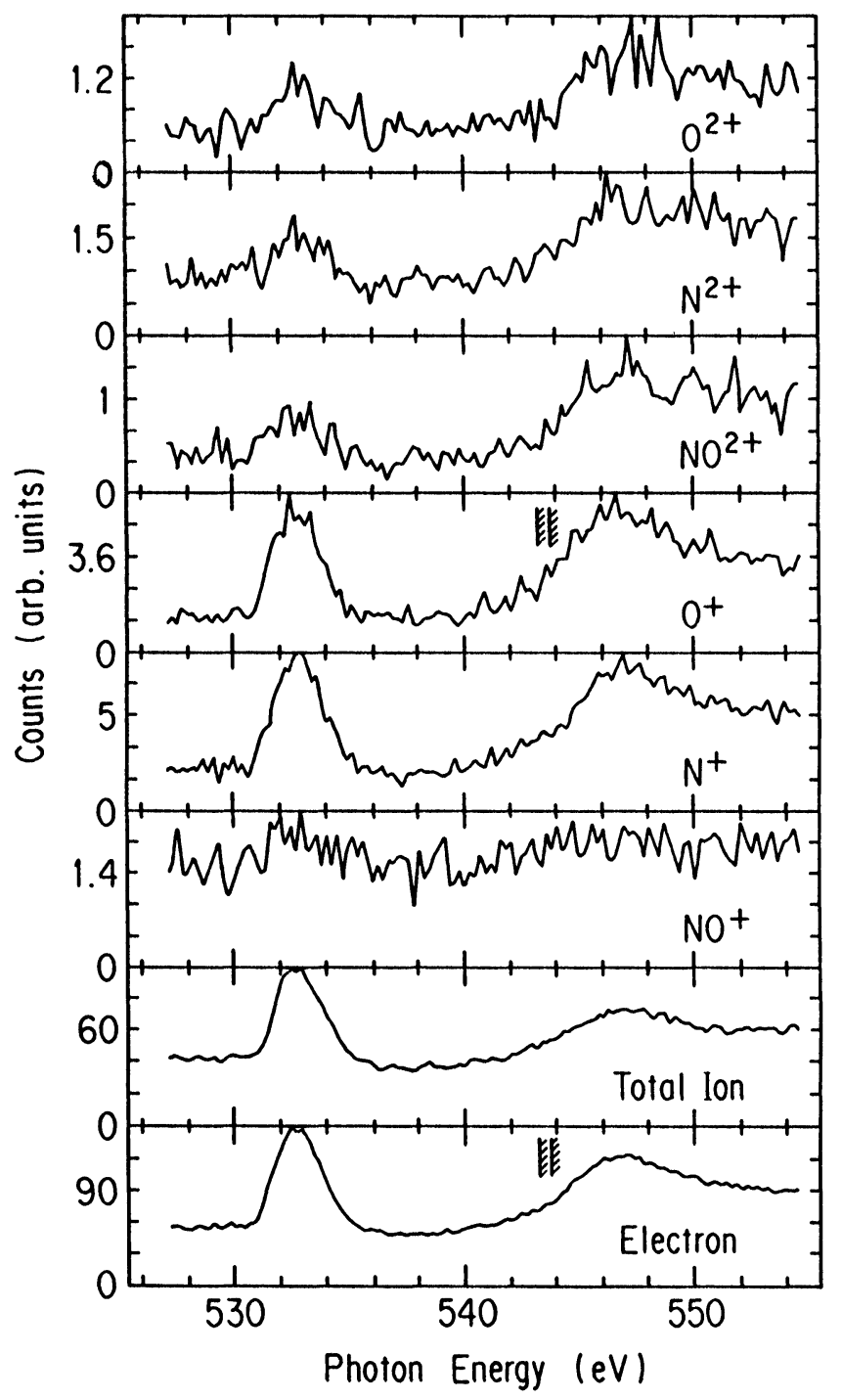

Figure 2 Yield spectra of individual ions produced from NO around the OK-edge, together with the total ion and the electron. Bars with hatching denote the ionization thresholds, ${ }^{3} \Pi$ at $543.3 \mathrm{eV}$ and ' $\Pi$ at $543.8 \mathrm{eV}$. 
photoabsorption spectrum observed at moderate resolution, which shows a broad peak ascribed to the $1 \sigma$ to $2 \pi$ transition at $532.6 \mathrm{eV}$ and a broad intensity increase composed of the OK-shell ionization and the $\sigma$-type shape resonance (around $546 \mathrm{eV}){ }^{11,14,15}$ The parent molecular ion $\mathrm{NO}^{+}$has low yields over this energy range and exhibits a slight intensity increase near $533 \mathrm{eV}$. The yield spectra of $\mathrm{N}^{+}$and $\mathrm{O}^{+}$ have profiles close to the electron curve. The yield curves of $\mathrm{N}^{2+}$ and $\mathrm{O}^{2+}$ essentially show the same feature as the electron, but the intensities around the shape resonance are higher than the broad structures at the $1 \sigma$ to $2 \pi$ transition, although the measured data scatter considerably. The intensity around the shape resonance in the $\mathrm{NO}^{2+}$ curve is higher than the broad structure near $533 \mathrm{eV}$. The general features, similarity and/or difference, among the yield spectra of individual ions in the OK-edge region are close to those in the NK-edge region.

\section{B. Kinetic energy distributions of fragment ions}

In order to obtain kinetic energies of the fragment ions, TOF spectra were measured with a weak electric field for ion extraction, $13 \mathrm{~V} / \mathrm{mm}$. The TOF spectra are shown for $\mathrm{N}^{+}, \mathrm{O}^{+}$and $\mathrm{NO}^{2+}$ at some photon energies in Fig. 3. As denoted in Fig. 3, the peak at the shortest flight time near $2.18 \mu$ s originates from the $\mathrm{N}^{+}$ion which is ejected toward the ion detector with some kinetic energy through the molecular dissociation. The most intense peak at $2.34 \mu \mathrm{s}$ comes from the $\mathrm{NO}^{2+}$ with thermal energy. The $\mathrm{N}^{+}$ion which is ejected toward the electron detector and extracted back to the ion detector contributes to the tail at the shorter time side of the strongest peak around $2.3 \mu \mathrm{s}$. The kinetic ions of $\mathrm{O}^{+}$generate the tail at the longer time side of the central peak and the peak at $2.5 \mu \mathrm{s}$ in the TOF spectrum. The structures of the spectra at the four photon energies are different from one another and can be approximately classified to two types. One is the spectrum that thermal and/or low kinetic energy $\mathrm{N}^{+}$ions yield the broad intense structure around $2.27 \mu$ s as well as the structures by kinetic $\mathrm{N}^{+}$ions, thermal $\mathrm{NO}^{2+}$ ions and kinetic $\mathrm{O}^{+}$ions. This type was measured for the photon energies inducing transitions of $1 \mathrm{~s}$ electrons to the $2 \pi$ orbital. The other is that thermal $\mathrm{N}^{+}$ions are produced with low probabilities. These spectra are for the photon energies corresponding to the ionizations of $1 \mathrm{~s}$ elections.

The measured TOF spectra were reproduced using the simulation calculation. ${ }^{11,23-28}$ An example of the fitted spectra is shown in Fig. 4. The dotts denote the experimental data, and the broken curves indicate the calculated TOF spectra for $\mathrm{NO}^{2+}$ with thermal energy and for $\mathrm{N}^{+}$and $\mathrm{O}^{+}$ions having several kinetic energies. The kinetic energies of $\mathrm{N}^{+}$used in the calculation were $0.36,0.81,1.44,2.25,3.21,4.41,5.76,7.27,9.0$, 10.83 and $12.96 \mathrm{eV}$ from the inner to the outer, and those of $\mathrm{O}^{+}$were $0.32,0.71$, $1.26,1.97,2.81,3.86,5.04,6.36,7.88,9.48$ and $11.34 \mathrm{eV}$. The solid curve represents the summation of the broken curves. This calculated curve has well reproduced the experimental results. The area surrounded with a broken curve gives the intensity of the ion having the corresponding kinetic energy, if this area is corrected for the collection efficiency of kinetic ions.

Figure 5 shows the kinetic energy distributions of $\mathrm{N}^{+}$and $\mathrm{O}^{+}$at some photon energies $(400,415,533$ and $547 \mathrm{eV})$. At the photoexcitation of $400 \mathrm{eV}(2 \sigma \rightarrow 2 \pi)$, 


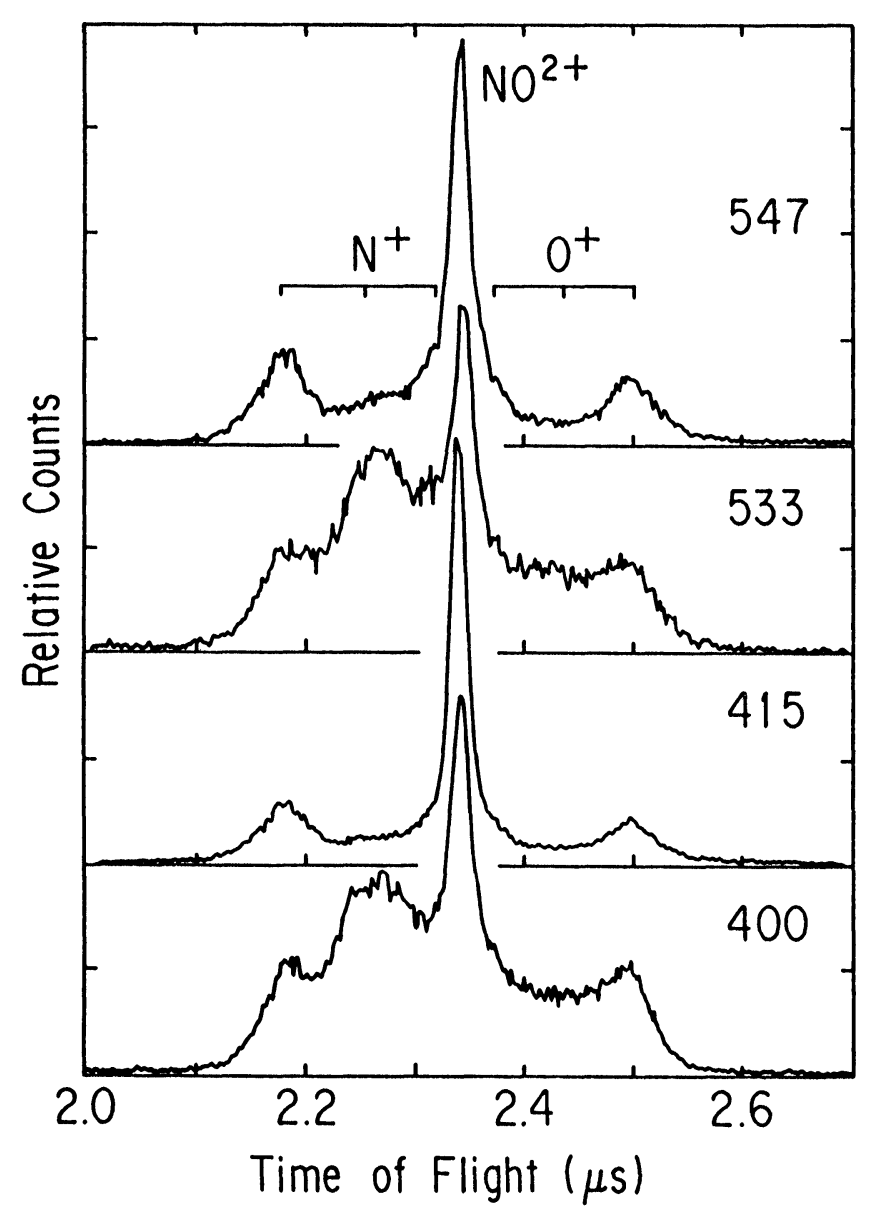

Figure 3 Detailed time-of-flight spectra of fragment ions produced from NO at the four photon energies corresponding to transitions of 1 s electron to the $2 \pi$ orbital and the continuum. Photon energies are listed at the right end.

$\mathrm{N}^{+}$ions having low kinetic energy $(<1.5 \mathrm{eV})$ were produced significantly, as expected from the TOF spectrum. The second maximum appears at about $4.5 \mathrm{eV}$. This distribution is similar to that for $\mathrm{N}^{+}$at $533 \mathrm{eV}$, which induces the transition from the $1 \sigma$ orbital to the $2 \pi$ orbital. The distribution for $\mathrm{N}^{+}$at $415 \mathrm{eV}$ (above the ionization thresholds of the $2 \sigma$ electron) is different from that at $400 \mathrm{eV}$. The fraction of low kinetic energy ions is very low at $415 \mathrm{eV}$. The distribution at $547 \mathrm{eV}$ (above the ionization thresholds of the $1 \sigma$ electron) is similar to that at $415 \mathrm{eV}$, which shows a low intensity at lower kinetic energies and a maximum near $4.5 \mathrm{eV}$. Going to profiles of $\mathrm{O}^{+}$kinetic energy distributions shown in the right hand side of Fig. 5, the dependence of the distributions on the photon energy is similar to that for $\mathrm{N}^{+}$ions. The maximum is seen at about $4 \mathrm{eV}$ in the distribution curve at every energy, and considerable intensities were obtained at lower kinetic energies only for the photoexcitation to the $2 \pi$ orbital ( $400 \mathrm{eV}$ and $533 \mathrm{eV}$ ). The energy position for the maximum 


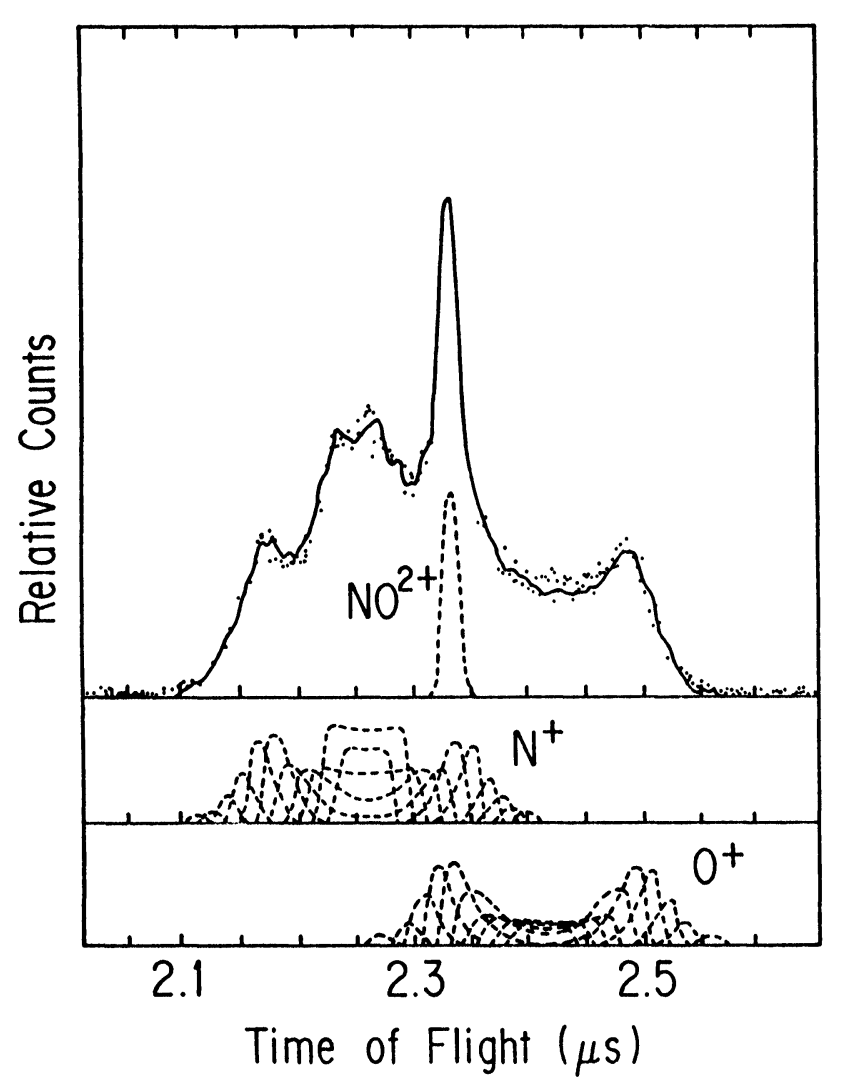

Figure 4 Calculated time-of-flight spectra of fragment ions at a photon energy of $400 \mathrm{eV}$ in comparison with the observed data. Broken curves denote the calculated TOF spectra for ions having a certain kinetic energy (see the text). Solid curve indicates the summation of the broken curves. Dotts are experimental data.

intensity is slightly lower for $\mathrm{O}^{+}$than that for $\mathrm{N}^{+}$, probably because of the mass difference of two elements. The intensities at lower kinetic energies for $\mathrm{O}^{+}$are smaller than those for $\mathrm{N}^{+}$at the photoexcitation to the $2 \pi$ orbital. This finding suggests that a singly charged molecular ion decomposes to $\mathrm{N}^{+}+\mathrm{O}$ with a higher probability than that to $\mathrm{N}+\mathrm{O}^{+}$because a considerable fraction of deexcitation pathways yield a singly charged molecule from inner-shell excited neutral states $\left(1 \mathrm{~s}^{1} 2 \pi^{2}\right)$.

The kinetic energy distributions of doubly charged atomic ions, $\mathrm{N}^{2+}$ and $\mathrm{O}^{2+}$, are shown for the four photon energies in Fig. 6. The $\mathrm{N}^{2+}$ distributions are similar to one another, but the energy position of the maximum intensity moves from about $6 \mathrm{eV}$ at the photon energy of $400 \mathrm{eV}$ to about $9 \mathrm{eV}$ at $547 \mathrm{eV}$. The feature for $\mathrm{O}^{2+}$ distributions is not much different from that for $\mathrm{N}^{2+}$. The distributions exhibit broad structures centered at 7-10 eV. This finding indicates a clear contrast to the feature of the distributions for the singly charged atomic ion $\mathrm{N}^{+}$, which represented a distinct difference between the various photoexcited states. 


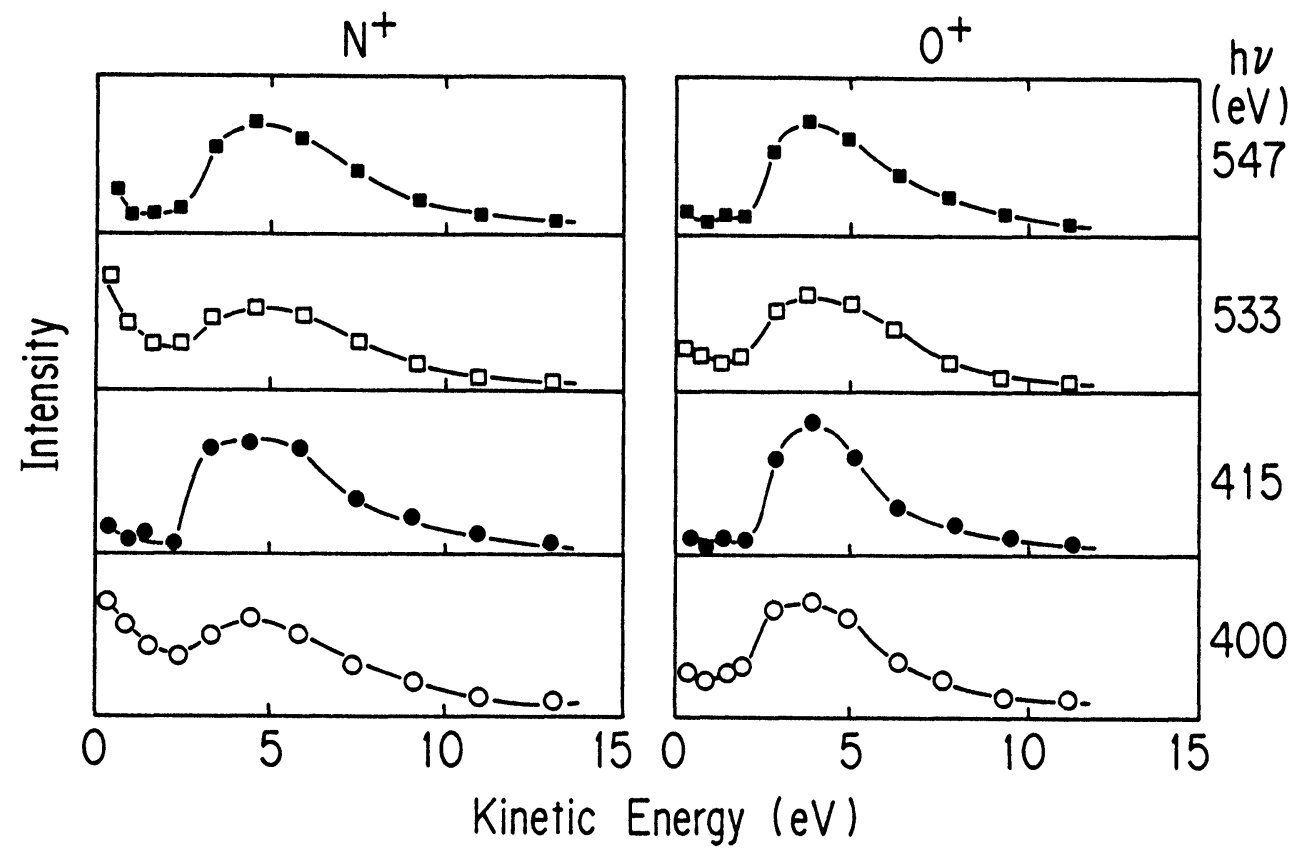

Figure 5 Kinetic energy distributions of $\mathrm{N}^{+}$and $\mathrm{O}^{+}$produced from $\mathrm{NO}$ at the four photon energies $(400,415,533$ and $547 \mathrm{eV})$. Photon energies are listed at the right end.

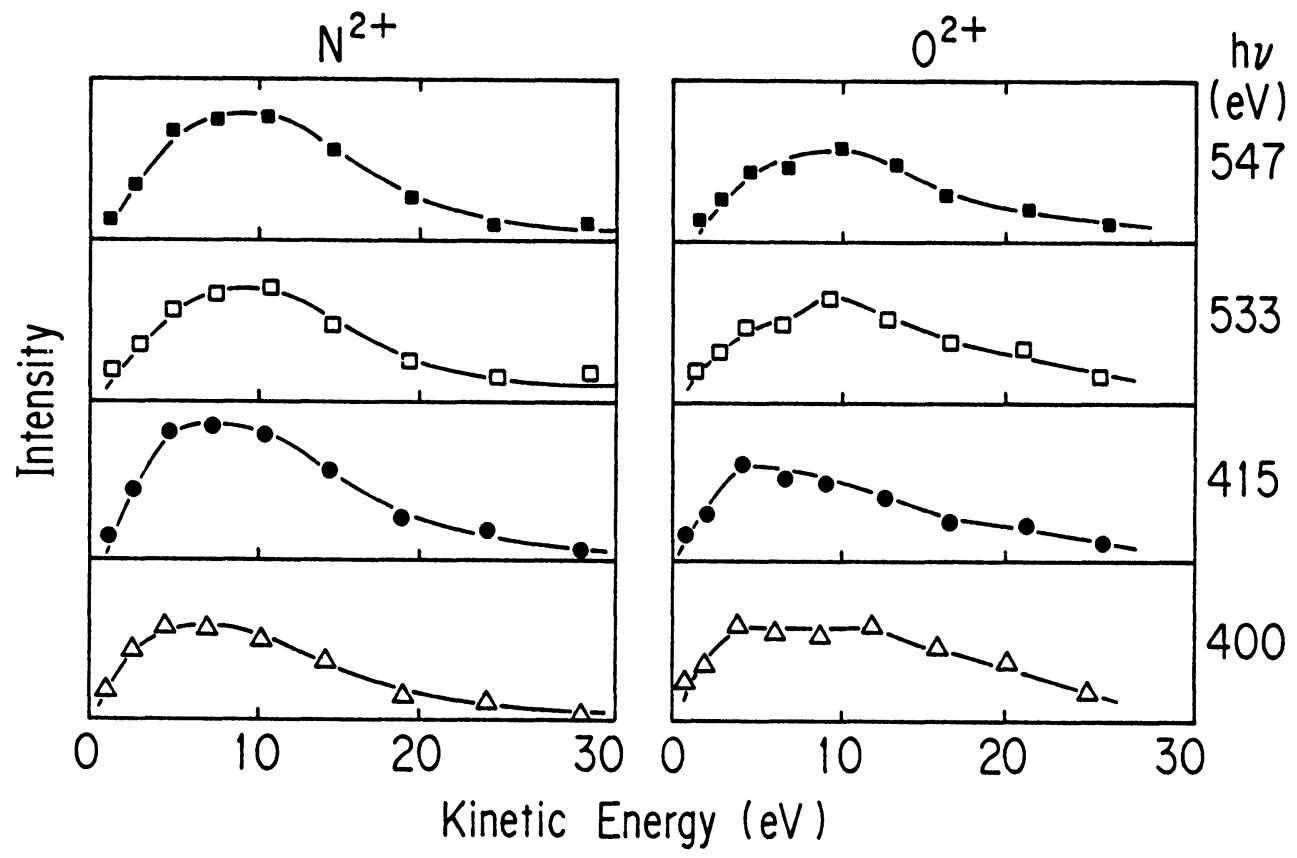

Figure 6 Kinetic energy distributions of $\mathrm{N}^{2+}$ and $\mathrm{O}^{2+}$ produced from $\mathrm{NO}$ at the four photon energies $(400,415,533$ and $547 \mathrm{eV})$. Photon energies are listed at the right end. 


\section{Dissociation pathways}

The molecule NO with a core-hole is supposed to emit electrons through an Augertype transition and then the highly excited molecular ion usually decomposes into some fragments as follows.

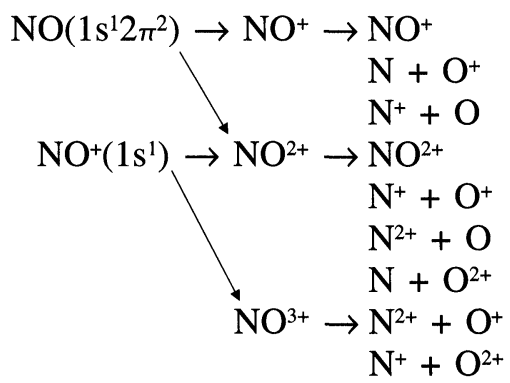

$9.3 \mathrm{eV})$
$20.1 \mathrm{eV})$
$21.0 \mathrm{eV})$
$38.5 \mathrm{eV})$
$34.6 \mathrm{eV})$
$50.6 \mathrm{eV})$
$55.2 \mathrm{eV})$
$64.2 \mathrm{eV})$
$69.7 \mathrm{eV})$

In these deexcitation schemes, broken arrows indicate the transition pathways which have not been understood to occur frequently. Energy values measured from the ground state of NO are listed in the right end when the formed species exist in their ground states. ${ }^{29,30}$

Table 1 Branching ratios of ions produced from NO at several photon energies.

\begin{tabular}{lllllllll}
\hline $\begin{array}{l}\text { Photon } \\
\text { Energy } \\
(\mathrm{eV})\end{array}$ & $\begin{array}{l}\text { Excited } \\
\text { State }\end{array}$ & $\mathrm{NO}^{+}$ & $\mathrm{NO}^{2+}$ & $\mathrm{N}^{+}$ & $\mathrm{O}^{+}$ & $N^{2+}$ & $\mathrm{O}^{2+}$ & \\
\hline 400 & $2 \sigma^{1} 2 \pi^{2}$ & 5 & 1 & 44 & 40 & 4 & 5 & \\
415 & $2 \sigma^{1} 2 \pi^{1}$ & 0 & 5 & 41 & 37 & 11 & 6 & \\
533 & $1 \sigma^{1} 2 \pi^{2}$ & 5 & 1 & 42 & 38 & 7 & 7 & \\
546 & $1 \sigma^{1} 2 \pi^{1}$ & 0 & 2 & 44 & 37 & 10 & 7 & $($ a) \\
80 & valence $^{-1}$ & 50.2 & 2.8 & 31.7 & 15.2 & & & \\
280 & valence $^{-1}$ & 73 & 0.6 & 11.6 & 14.9 & $<0.3$ & $<0.3$ & (b) \\
930 & $1 \sigma^{2} 2 \pi^{1}$ & 5.6 & 2.3 & 33 & 36 & 12.6 & 10 & (b) \\
\hline
\end{tabular}

(a): Data by a simulated photoionization technique (Ref. 4).

(b): Data using filtered characteristic X-rays (Ref. 8).

The branching ratio of ion intensity is presumed to be useful for discussing the dissociation pathways from different core-hole states. Table 1 lists the branching ratios at several photon energies, where the contribution from the ionization of only valence electrons for the NK-shell excitation and that of valence and the NK-shell electrons for the OK-shell excitation were subtracted. The data previously obtained with a simulation technique of photoionization and with filtered characteristic X-rays were included in Table 1.,8 The most intense ion is the singly charged nitrogen atomic ion $\left(\mathrm{N}^{+}\right)$at all photon energies. The second is the $\mathrm{O}^{+}$ion, and the doubly charged $\mathrm{N}^{2+}$ has a higher intensity above the ionization thresholds than the doubly charged oxygen ion $\mathrm{O}^{2+}$. The singly charged parent molecular ion is formed only at the transition energies of a $1 \mathrm{~s}$ electron to the $2 \pi$ orbital. The doubly charged molecular ion $\mathrm{NO}^{2+}$ is produced appreciably. The previous data below the excitation 
energy of the $2 \sigma$ electron differ considerably from each other although the photon energies used were much different. ${ }^{4,8}$ The present result at $400 \mathrm{eV}$ is nearer to the branching ratios by Iida et al. than to the relative abundances by Carlson and Krause, when the data with no valence subtraction are considered. Above the ionization threshold of the OK-shell, the previous datum at $930 \mathrm{eV}$ is close to the present result at $546 \mathrm{eV}$, where the branching ratios of the singly charged atomic ions are higher than $30 \%$ and those of the doubly charged ones are about $10 \%$.

It is important to examine the Auger electron spectrum closely because this spectrum provides energies of doubly charged molecular ions. Auger electron spectra were reported by some groups, which showed small transition intensities through autoionization from $\left(1 \mathrm{~s}^{1} 2 \pi^{2}\right)$ states as well as the normal Auger transitions. ${ }^{19-22}$ Lower states of $\mathrm{NO}^{2+}$ derived from $5 \sigma^{1} 2 \pi^{0}, 1 \pi^{3} 2 \pi^{0}$ and $4 \sigma^{1} 2 \pi^{0}$ configurations, which are positioned at about $38.5,40.0,43.0 \mathrm{eV}$, respectively, are populated more efficiently through N1s core-hole states than through O1s core-hole states. This is because the $2 \pi$ orbital is localized at the nitrogen atom. The states around $45 \mathrm{eV}-55 \mathrm{eV}$ are most populated through Auger transitions from both N1s hole and O1s hole states and their configurations are assigned to be $5 \sigma^{1} 1 \pi^{3}, 5 \sigma^{0}, 1 \pi^{2}$, and $4 \sigma^{1} 1 \pi^{3}$. In these instances, the electron in the $2 \pi$ orbital does not participate in the Auger transition. The Auger decay generating final states around $60 \mathrm{eV}-77 \mathrm{eV}$ produces slightly different populations of $\mathrm{NO}^{2+}$ states in $\mathrm{N} 1 \mathrm{~s}$ hole and $\mathrm{O} 1 \mathrm{~s}$ hole states. The main configurations for these final states are $4 \sigma^{0}, 3 \sigma^{1} 5 \sigma^{1}, 3 \sigma^{1} 1 \pi^{3}$, and $3 \sigma^{1} 4 \sigma^{1}$. The intensities in this energy region in the N1s and O1s spectra, as a whole, are very close to each other, although the electron in the $3 \sigma$ orbital, mainly localized at the oxygen atom, dominantly participates in the Auger transition. This phenomenon comes from the configuration interaction effect occurring among the states composed of the inner valence orbitals, as previously pointed out. ${ }^{4,20}$ The population of states above $80 \mathrm{eV}$ is low through the Auger decay, which correspond to the configurations of $3 \sigma^{1} 4 \sigma^{1}$ and $3 \sigma^{0}$. The broad structures can be seen in the O1s spectrum but few peaks are observed in the N1s spectrum.

Bearing in mind the population of $\mathrm{NO}^{2+}$ states through Auger transitions, let us examine first the kinetic energy distributions of fragment ions above the ionization thresholds. The maximum at $3-6 \mathrm{eV}$ for $\mathrm{N}^{+}$and that at $4 \mathrm{eV}$ for $\mathrm{O}^{+}$probably originate from the dissociation pathways in which the states of $\mathrm{NO}^{2+}$ around $45-55 \mathrm{eV}$ predominantly go to the ground states of $\mathrm{O}^{+}\left({ }^{4} \mathrm{~S}\right)$ and $\mathrm{N}^{+}\left({ }^{3} \mathrm{P}\right)$ and/or low excited states. This interpretation is based on the fact that the difference in energy between the intermediate states and the final products turns into the kinetic energy of the products if internal excitation or photon emission does not occur. The states of $\mathrm{NO}^{2+}$ around $40 \mathrm{eV}$ turn into $\mathrm{N}^{+}+\mathrm{O}^{+}$having low kinetic energy and some of them remain as a doubly charged molecular ion. The $\mathrm{NO}^{2+}$ states between $60 \mathrm{eV}$ and $80 \mathrm{eV}$ possibly decompose to $\mathrm{N}^{2+}+\mathrm{O}$ and $\mathrm{N}+\mathrm{O}^{2+}$. This decomposition gives kinetic energies of $7-15 \mathrm{eV}$ to $\mathrm{N}^{2+}$ and $\mathrm{O}^{2+}$ when product fragments are in their ground states. The highest $\mathrm{NO}^{2+}$ states presumably provide higher kinetic energies to the doubly charged fragment ions. A part of these states possibly connect to the dissociation pathways into $\mathrm{N}^{2+}+\mathrm{O}^{+}$and $\mathrm{N}^{+}+\mathrm{O}^{2+}$, as given by Eqns. (8) and (9). The branching ratio of $\mathrm{NO}^{2+}$ above the ionization thresholds of $\mathrm{N} 1 \mathrm{~s}$ electron differs from that of $\mathrm{O} 1 \mathrm{~s}$ ioniza- 
tion, as shown Table 1. This finding supports that some states of $\mathrm{NO}^{2+}$ around $40 \mathrm{eV}$ do not dissociate but survive because the intensity of these states populated through the N1s Auger decay is much higher than that of the O1s Auger decay. The branching ratio of $\mathrm{N}^{2+}$ slightly decreased with changing the photon energy from $415 \mathrm{eV}$ to $546 \mathrm{eV}$ but that of $\mathrm{O}^{2+}$ increased. This finding is consistent with the result that the relative population of higher $\mathrm{NO}^{2+}$ states via the Auger decay is slightly smaller at the N1s Auger transition than the counterpart of O1s hole.

At the excitation to the $2 \pi$ orbital, photon energies of $400 \mathrm{eV}$ and $533 \mathrm{eV}$, deexcitation of electronic states usually takes place to produce singly ionized molecular ion. ${ }^{22}$ Some of the produced states have one hole in the valence orbitals except the $2 \pi$ orbital like a configuration of $5 \sigma^{1} 2 \pi^{1}$ or no electron in the latter orbital $\left(2 \pi^{0}\right)$. The others have two electrons in the $2 \pi$ orbital and two holes in the valence orbitals, e.g. $5 \sigma^{1} 1 \pi^{3} 2 \pi^{2}$. The former states are positioned mainly at $9.3-23.3 \mathrm{eV}$. The latter group of ion states lie probably above $23 \mathrm{eV}$, as shown in the recent work using an electron-electron coincidence technique. ${ }^{22}$ Many of the former states can survive as the parent molecular ion because the threshold energy of the dissociation is $20.1 \mathrm{eV}$. Small fraction of them turn into $\mathrm{N}+\mathrm{O}^{+}$and $\mathrm{N}^{+}+\mathrm{O}$, which have low kinetic energies. The kinetic energy distributions of $\mathrm{N}^{+}$and $\mathrm{O}^{+}$at the photon energy of the excitation to the $2 \pi$ orbital (Fig. 5) have significant intensities in very low energy region. Lower energy states of the latter group, states below $34 \mathrm{eV}$, decompose to $\mathrm{N}+\mathrm{O}^{+}$and $\mathrm{N}^{+}$ $+\mathrm{O}$, in which the individual fragments have a kinetic energy of $1-6 \mathrm{eV}$ on the basis of the calculation of energy difference between the intermediate states and final products. The states of $\mathrm{NO}^{+}$above $35 \mathrm{eV}$ can emit another electron and contribute to the ion-pair formation of $\mathrm{N}^{+}+\mathrm{O}^{+}$. These ions possibly have appreciable kinetic energies. If the $\mathrm{NO}^{+}$states are positioned above $50 \mathrm{eV}$, these states can produce a doubly charged fragment ion, accompanied by a second electron emission.

However, the relative population of $\mathrm{NO}^{+}$states derived from autoionization of $\left(1 \mathrm{~s}^{1} 2 \pi^{2}\right)$ states is concentrated into the energy region of $16-29 \mathrm{eV}$, according to the study by Carroll and Thomas. ${ }^{22}$ Based on that population and a consideration of energy levels, the excitation to the $2 \pi$ orbital is supposed to provide a large branching ratio for $\mathrm{NO}^{+}$, which is relatively close to those of $\mathrm{N}^{+}$and $\mathrm{O}^{+}$, a maximum at the low energy region in the kinetic energy distributions of $\mathrm{N}^{+}$and $\mathrm{O}^{+}$, and a very low branching ratios for $\mathrm{N}^{2+}$ and $\mathrm{O}^{2+}$. This supposition does not agree with the present results. This fact postulates necessity of another pathway of deexcitation from the $\left(1 \mathrm{~s}^{1} 2 \pi^{2}\right)$ states. This pathway is possibly an Auger shake-off (double Auger) process, in which the K-shell hole is filled with ejection of two valence electrons. This process cannot be observed using the coincidence technique by Carroll and Thomas because the emitted electrons are able to share the kinetic energy in a variety of ratios. The produced states of $\mathrm{NO}^{2+}$ are probably similar to those by the normal Auger transition. These intermediate states yield a maximum at a relatively high energy region of the kinetic energy distributions of $\mathrm{N}^{+}$and $\mathrm{O}^{+}$and a considerable magnitude of the branching ratios for $\mathrm{N}^{2+}$ and $\mathrm{O}^{2+}$. In instances of $\mathrm{N}_{2}$ and $\mathrm{O}_{2}$, Auger shake-off transitions happen with a probability of $10 \%$ or so in photoexcitation of the 1 s electron to the $\pi^{*}$ orbital. ${ }^{23,24,26}$ The branching ratios of $\mathrm{NO}^{2+}$ at $400 \mathrm{eV}$ and $415 \mathrm{eV}$ are $1 \%$ and $5 \%$, respectively, as shown in Table 1 . This result suggests that the probability ratio of 
the Auger shake-off process is near to about $20 \%$ at $400 \mathrm{eV}$. The corresponding branching ratios of $\mathrm{NO}^{2+}$ at the $\mathrm{O} 1 \mathrm{~s}$ excitation are $1 \%$ and $2 \%$. This finding suggests that the probability ratio of the shake-off process is higher at the 01 s excitation than the N1s excitation. This suggestion is supported by the finding on the branching ratios and kinetic energy distributions of $\mathrm{N}^{2+}$ and $\mathrm{O}^{2+}$ at $533 \mathrm{eV}$. These ratios increased slightly and the energy position giving the maximum intensity shifted to higher energy at $533 \mathrm{eV}$, compared to those at $400 \mathrm{eV}$.

\section{Summary}

Fragment ions produced from photoexcitation of NO in the soft X-ray region have been observed using the TOF mass spectrometer. Detailed yield spectra for individual ions were measured in the regions of NK-shell and OK-shell ionization edges. Kinetic energy distributions of these ions were estimated and discussed for clarifying dissociation pathways of $\mathrm{K}$-shell excited molecule. This discussion suggests that many of the product ions exist in their ground or lower excited states and that a considerable fraction of deexcitation from the $\left(1 \mathrm{~s}^{1} 2 \pi^{2}\right)$ states yield doubly charged species through emission of two electrons, e.g. Auger shake-off process.

\section{Acknowledgements}

The authors wish to express their sincere thanks to the members of the accelerator group of the Electrotechnical Laboratory for providing synchrotron radiation. One of us (I. H. S.) is grateful to Prof. Soji Tsuchiya for kind guidance and fruitful advice in the early stage as a researcher.

\section{References}

1. H. Hertz, H. W. Jochims, H. Schenk and W. Sroka, Chem. Phys. Lett., 29572 (1973).

2. F. H. Dorman and J. D. Morrison, J. Chem. Phys., 35575 (1961).

3. P. C. Kronebusch and J. Berkowitz, Int. J. Mass Spectrom. Ion Phys., 22283 (1976).

4. Y. Iida, F. Carnovale, S. Daviel and C. E. Brion, Chem. Phys., 105211 (1986).

5. J. A. R. Samson, T. Masuoka and P. N. Pareek, J. Chem. Phys., 835531 (1985).

6. P. M. Hierl and J. L. Franklin, J. Chem. Phys., 473154 (1967).

7. J. M. Curtis and R. K. Boyd, J. Chem. Phys., 812991 (1984).

8. T. A. Carlson and M. O. Krause, J. Chem. Phys., 563206 (1972).

9. T. Masuoka, Phys. Rev., A48 1955 (1993).

10. T. Masuoka, J. Chem. Phys., 1006422 (1994).

11. N. Saito and I. H. Suzuki, Phys. Rev., A43 3662 (1991).

12. N. Kosugi, J. Adachi, E. Shigemasa and A. Yagishita, J. Chem. Phys., 978842 (1992).

13. G. Remmers, M. Domke, A. Puschmann, T. Mandel, G. Kaindl, E. Hudson and D. A. Shirley, Chem. Phys. Lett., 214241 (1993).

14. M. Tronc, G. C. King and F. H. Read, J. Phys., B13 999 (1980).

15. G. R. Wight, C. E. Brion and M. J. Van der Wiel, J. Electron Spectrosc. Relat. Phenom., 1457 (1972).

16. W. Eberhardt, T. K. Sham, R. Carr, S. Krummacher, M. Strongin, S. L. Weng and D. Wesner, Phys. Rev. Lett., 501038 (1983).

17. S. Nagaoka, J. Oshita, M. Ishikawa, T. Masuoka and I. Koyano, J. Phys. Chem., 971488 (1993).

18. I. H. Suzuki, J. D. Bozek and N. Saito, Chem. Phys., 18281 (1994). 
19. W. E. Moddeman, T. A. Carlson, M. O. Krause, B. P. Pullen, W. E. Bull and G. K. Schweitzer, J. Chem. Phys., 552317 (1971).

20. H. Agren, J. Chem. Phys., 751267 (1981).

21. L. G. M. Pettersson, L. Karlsson, M. P. Keane, A. N. de Brito, N. Correia, M. Larsson, L. Brostrom, S. Mannervik and S. Svensson, J. Chem. Phys., 964884 (1992).

22. T. X. Carroll and T. D. Thomas, J. Chem. Phys., 97894 (1992).

23. I. H. Suzuki and N. Saito, J. Chem. Phys., 915324 (1989).

24. N. Saito and I. H. Suzuki, J. Chem. Phys., 915329 (1989).

25. N. Saito and I. H. Suzuki, Phys. Rev. Lett., 612740 (1988).

26. N. Saito and I. H. Suzuki, Int. J. Mass Spectrom. Ion Proces., 8261 (1988).

27. J. D. Bozek, N. Saito and I. H. Suzuki. J. Chem. Phys., 100393 (1994).

28. N. Saito and I. H. Suzuki, J. Phys., B22 3973 (1989).

29. S. Bashkin and J. O. Stoner, Jr., Atomic Energy Levels and Grotrian Diagrams, Vol. 1, North-Holland (Amsterdam, 1975).

30. J. Appell, J. Durup, F. C. Fehsenfeld and P. Fournier, J. Phys., B6 197 (1973). 